\title{
MATERNAL HOMOCYSTEINE IN ASSOCIATION WITH BIRTH WEIGHT: SYSTEMATIC REVIEW AND META-ANALYSIS
}

\author{
M. Hogeveen ${ }^{1}$, H.J. Blom ${ }^{2}$, M. den Heijer ${ }^{3}$
}

${ }^{1}$ Paediatrics and Neonatology, Radboud University Nijmegen, Medical Centre, Nijmegen, ${ }^{2}$ Metabolic Unit, Dept. of Clinical Chemistry, VU University Medical Centre Amsterdam, Amsterdam, ${ }^{3}$ Dept. of Epidemiology and Biostatistics, Dept. of Endocrinology, Radboud University Nijmegen, Medical Centre, Nijmegen, The

Netherlands

Background and aims: Low birth weight (LBW), as a proxy for fetal growth, is associated with increased neonatal morbidity and mortality but also with life-long consequences such as poor cognitive function and cardiovascular diseases. Maternal total homocysteine concentrations (tHcy) have been linked to a wide range of adverse pregnancy outcomes and could possibly influence birth weight. We performed a systematic review and meta-analysis on the association of maternal tHcy and birth weight.

Methods: An electronic literature search revealed 78 abstracts. Studies were eligible if information on maternal tHcy, birth weight and the possible association between maternal tHcy and birth weight was available. Effect estimates were converted to odds ratios with a cut-off level of birth weight $<10^{\text {th }}$ percentile for gestational age (SGA) and maternal tHcy $>90^{\text {th }}$ percentile.

Results: The search yielded 19 studies for analysis, consisting of 21,326 individuals. Pooled analysis resulted in a crude OR of $1.30[1.14 ; 1.49]$ (relative risk to get an infant born SGA when maternal tHcy is $>90^{\text {th }}$ percentile). This estimate corresponds to $-43[-19 ;-56] \mathrm{g}$ for $1 \mathrm{sd}$ increase in maternal tHcy. Adjustment for known confounders was not possible but a tendency to decreased strength of association was observed in studies after adjustment for strong determinants.

Conclusion: Higher maternal tHcy concentrations are associated with increased risk for being born SGA. Adjustment for possible confounders was performed in some but not all studies. The small estimated birth weight difference might be of little clinical relevance for the individual newborn, however, it could be of greater importance on population level. 